\title{
Three-dimensional Ultrasonographic Presentation of Micrognathia
}

\author{
Wesley Lee, MD, Beverley McNie, Tinnakorn Chaiworapongsa, MD, \\ Giancarlo Conoscenti, MD, Karim D. Kalache, MD, Ivana M. Vettraino, MD, \\ Roberto Romero, MD, Christine H. Comstock, MD
}

\begin{abstract}
Objective. To present the variable appearance of micrognathia in fetuses by three-dimensional ultrasonography and to describe practical methods for analysis of these volume data. Methods. Threedimensional multiplanar imaging and surface-rendering techniques were used to show various syndromes and diagnostic approaches for the evaluation of fetal micrognathia. Results. Nine cases of fetal micrognathia are presented. Orthogonal multiplanar views were used to obtain a midsagittal facial profile. Examples of micrognathia include 3 cases of Pierre Robin sequence, cerebrocostomandibular syndrome, Cornelia de Lange syndrome, and hypochondrogenesis. Diagnostic pitfalls for micrognathia are also shown. Conclusions. Three-dimensional multiplanar imaging increases the likelihood that a true midline sagittal view of the facial profile is being analyzed. Surface rendering provides another way to qualitatively evaluate the fetal chin from different viewing perspectives. Three-dimensional ultrasonographic methods are useful adjuncts to the preliminary diagnostic impression from two-dimensional ultrasonography. Key words: fetus; micrognathia; prenatal diagnosis; three-dimensional ultrasonography.
\end{abstract}

Abbreviations

3D, three-dimensional; 3DUS, three-dimensional ultrasonography; 2D, two-dimensional; 2DUS, two-dimensional ultrasonography

\section{Introduction}

Micrognathia refers to a facial malformation that is characterized by a small chin. The Online Mendelian Inheritance in Man database at the National Library of Medicine $^{1}$ includes 211 genetic conditions with this finding. Affected fetuses are at increased risk for an abnormal karyotype. ${ }^{2,3}$ An underdeveloped chin may even be severe enough to cause hydramnios in the fetus or respiratory obstruction after delivery. Its presence may even complicate infant feeding and will typically require 1 or more surgical repairs. Here we present images from several fetuses with micrognathia who were examined with three-dimensional ultrasonography (3DUS).

\section{Materials and Methods}

Volume data were acquired from fetuses, including 8 with confirmed cases of suspected micrognathia (Voluson 530 and 730; GE Medical Systems, Milwaukee, 
WI). Each scan sweep took approximately 6 seconds and was usually performed as a sagittal scan across the fetal face with a $3-$ to $5-\mathrm{MHz}$ mechanical volume transducer. These data were stored on removable digital media for subsequent analysis (3D View software, version 1.0; GE Medical Systems). Orthogonal multiplanar images were reformatted from volume data. Postprocessing included adjustment of brightness, contrast, opacity, and transparency. Tissue contrast resolution was enhanced by addition of color filtering. Uninformative voxels were digitally removed by an image segmentation tool. Rendered images were composed (Photoshop 5.5; Adobe Systems Incorporated, San Jose, CA) for direct comparisons between multiplanar data and postnatal results (see Figs. 2, 3B, 4-6, 7B, 8, 9B, and 10). Color reference dots in the multiplanar views were enlarged for illustration purposes as well.

\section{Results}

Examples of fetal micrognathia and diagnostic pitfalls are presented in Figures 1-10.

\section{Discussion}

Prenatal diagnosis of micrognathia has traditionally relied on subjective interpretation of two-dimensional (2D) images that are mentally interpreted by the sonologist. ${ }^{2,12}$ Others have attempted to use mandibular measurements for this assessment. ${ }^{13-15}$ Paladini and coworkers ${ }^{15}$ applied a newborn parameter, the jaw index, to 262 fetuses without abnormalities between 12 and 37 weeks' gestation. ${ }^{16}$ Their population of 198 fetuses with malformations included 11 with micrognathia. The jaw index led to an improved micrognathia detection rate $(100 \%$ sensitivity and $98.1 \%$ specificity) compared with subjective evaluation of the facial profile $(72.7 \%$ sensitivity and $99.2 \%$ specificity). At present it is unclear whether 3DUS will enable us to improve our ability to measure mandibular size. ${ }^{17}$

Three-dimensional ultrasonography can provide additional information about the face in ways that are complementary to conventional 2D ultrasonography (2DUS). The most important advantage of 3DUS is its ability to display a true midsagittal plane of the fetal face. Diagnostic inaccuracies, however, can result from inappro-

Figure 1. A, Normal facial profile, 25 menstrual weeks (midline sagittal view). Three-dimensional multiplanar imaging is used to standardize orthogonal views of the face. Color reference dots represent the same anatomic location from all 3 viewing planes. By lining up the vertical scanning plane (white lines) from the coronal view (top left), it is possible to interactively display a true midsagittal facial profile (top right). The chin tapers to a relatively sharp apex. A rounded forehead also lines up with the most anterior portion of the chin. An axial view (bottom left) confirms that both orbits are symmetrically sectioned, as they should appear if the head is correctly oriented in a coronal plane. B, False micrognathia (oblique sagittal view). A coronal view of the face has now been rotated about $20^{\circ}$ (clockwise) to display an oblique sagittal profile of the same fetus. The corresponding axial view (bottom left) reveals an asymmetric appearance for both orbits, because the orange reference lines are not aligned along a true coronal plane (top left). Without interactive visualization of orthogonal planes by 3D multiplanar imaging, one could reach an erroneous diagnosis of micrognathia by misinterpreting an oblique sagittal profile (top right).

A

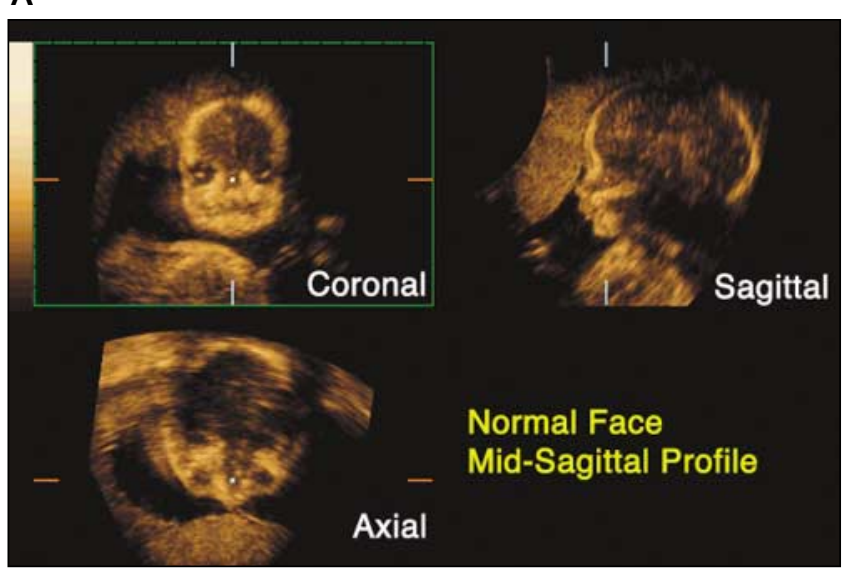

B

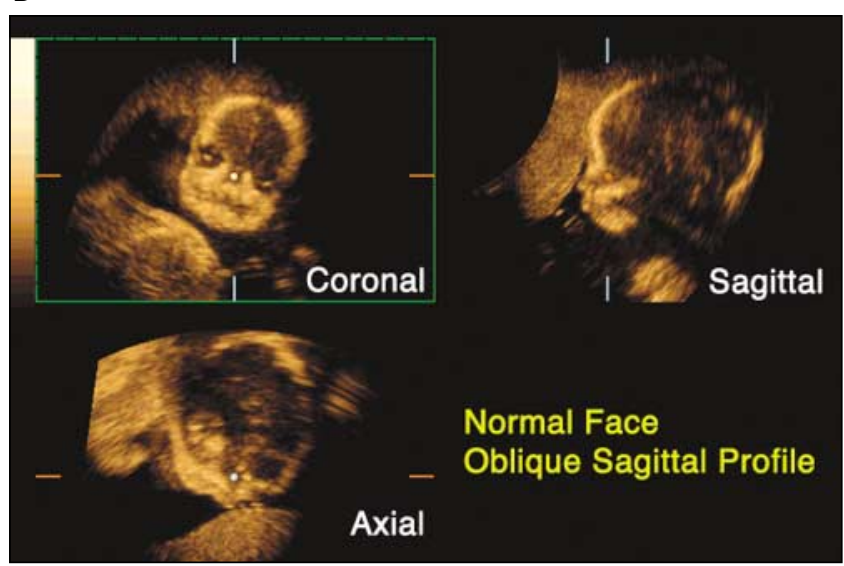


priate manipulation of multiplanar images (Fig. 1B) and rendered volume data (Fig. 5B). Careful examination of facial dysmorphology, such as cleft lip and palate, can provide important clues for genetic syndromes that are often associated with micrognathia. One of the uses of 3DUS surface rendering is to enable referring physicians to counsel prenatal patients accurately. Individuals who do not have specific training in diagnostic ultrasonography have a more difficult time with mentally formulating 3D reconstructions from standard 2D images. These types of 3D images are helpful for communicating with medical colleagues without this expertise, such as reconstructive surgeons and pediatricians. Finally, rendered displays of fetal surface anatomic features can be instrumental for counseling parents about suspected abnormalities.

Future investigations may eventually lead to improved diagnostic methods for evaluation of mandibular size and growth. Quantitative ultrasonographic parameters (e.g., mandibular area, volume, jaw index, and angles) have the potential for improving our ability to better characterize micrognathia, because standardized measurements are more likely to be obtained by $3 \mathrm{D}$ multiplanar imaging. Both qualitative and quantitative methods, however, may be necessary for optimal evaluation of micrognathia.

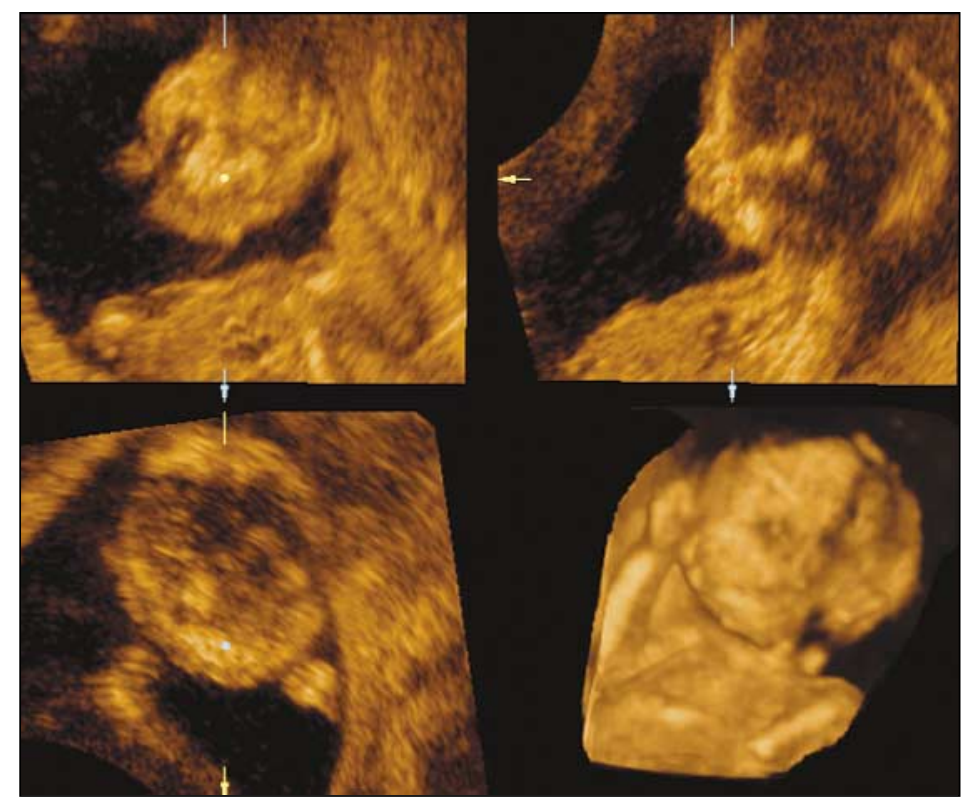

Figure 2. Pierre Robin sequence (case 1), 18.9 menstrual weeks. Pierre Robin sequence consists of micrognathia, cleft palate, or both. ${ }^{4} \mathrm{~A}$ small, rounded chin is shown in the midsagittal plane (top right). In an axial view (bottom left), a reference dot is inserted on the intact maxillary alveolar tooth buds, which suggest an intact anterior hard palate. ${ }^{5}$ One cannot exclude the presence of a posterior hard palate defect, because this condition does not usually involve the anterior hard palate.

Figure 3. Cerebrocostomandibular syndrome, 36.4 menstrual weeks. A, With this approach, a green cutting plane is placed across the rendered face (bottom right) to display a true midline sagittal view (top left). An axial view (top right) confirms normal maxillary tooth buds with an intact overlying upper lip (yellow arrow). A very small chin with marked polyhydramnios developed in this fetus by the late third trimester. Micrognathia can increase the amniotic fluid volume by preventing effective swallowing. Intubation failed after delivery, and the neonate required an emergent tracheostomy and mechanical ventilation. B, Facial profiles are examined from different angles by rotating the volume reconstruction. Occasionally, the chin may appear to be smaller because of posterior displacement of the mandible (i.e., retrognathia) or prominent cheeks (e.g., diabetes). Cerebrocostomandibular syndrome was diagnosed on the basis of micrognathia, a posterior cleft palate defect, and characteristic rib gap abnormalities (not shown) in the neonate..$^{6,7}$

A

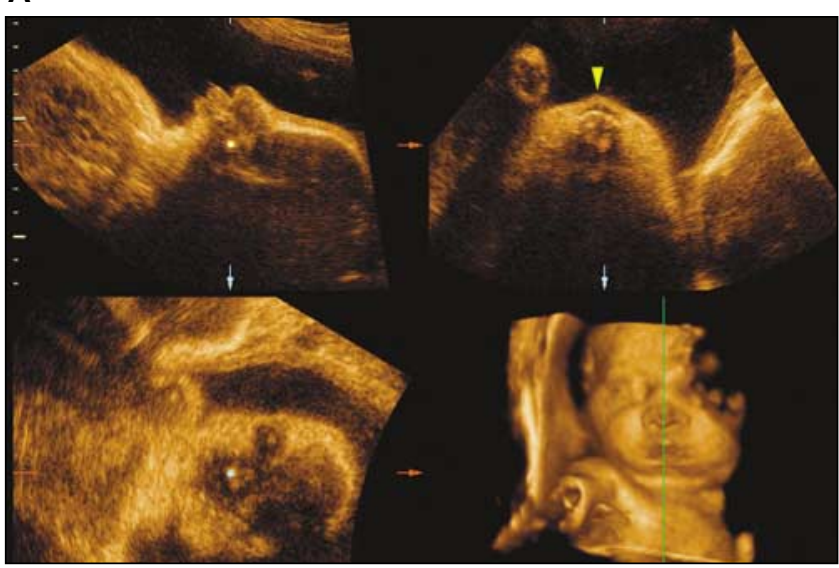

B

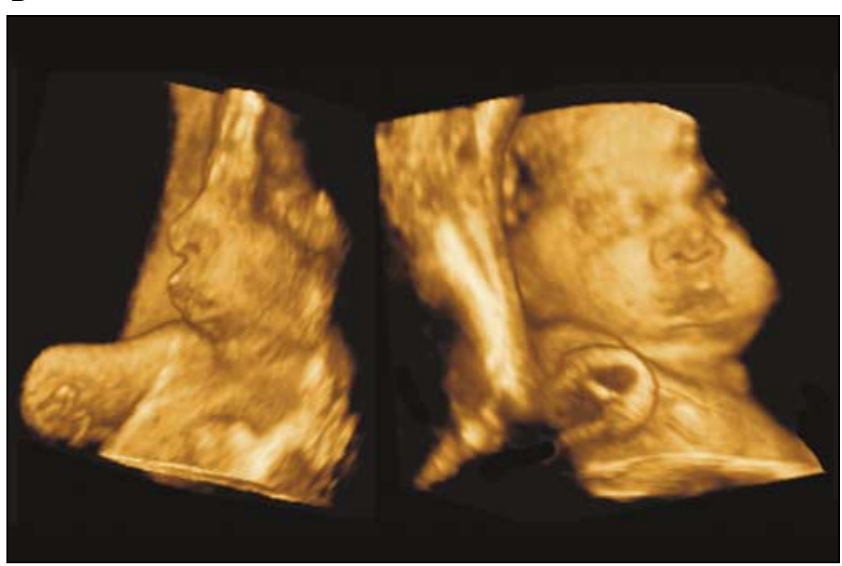




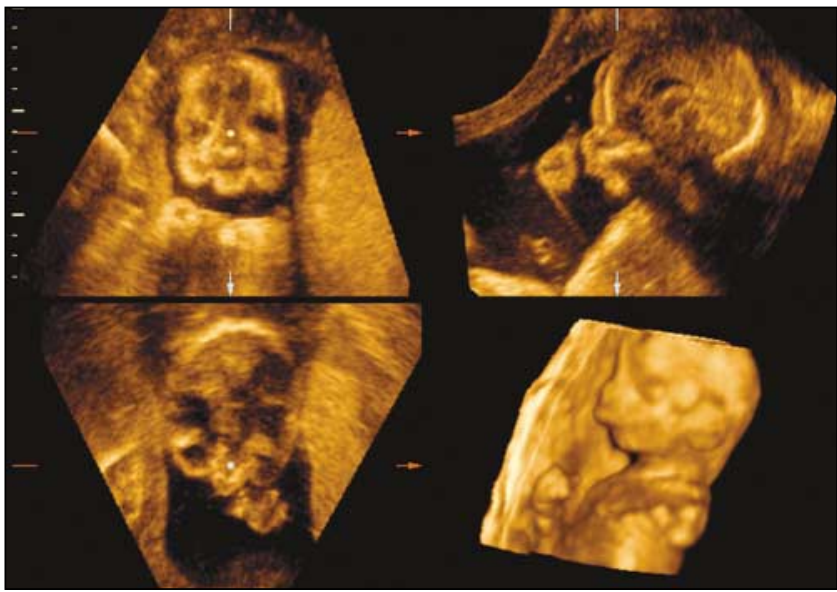

A

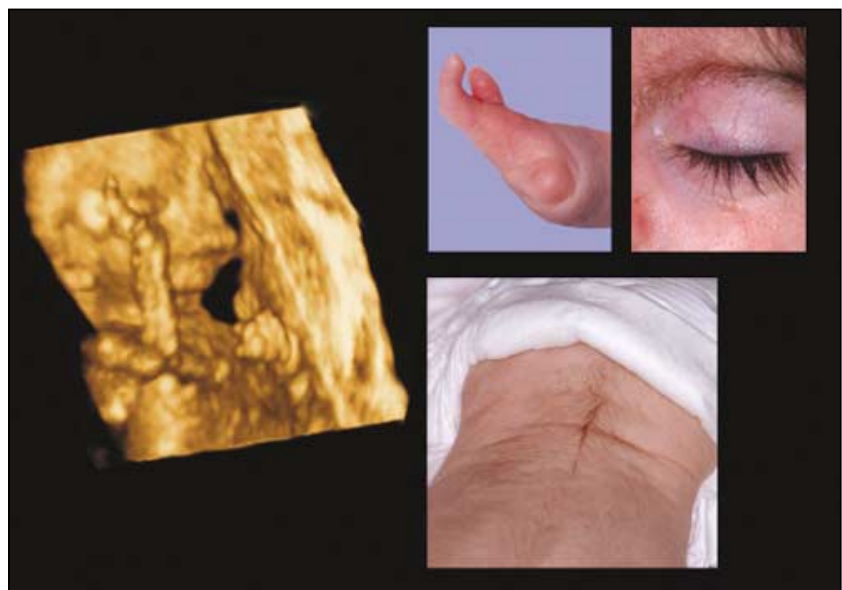

B

Figure 4. Cornelia de Lange syndrome, 19.9 menstrual weeks. A, This fetus has an underdeveloped chin with tetralogy of Fallot (not shown), intrauterine growth restriction, and an abnormal right hand. B, The right hand has a shortened thumb with 2 malformed digits. Cornelia de Lange syndrome was considered as a possible prenatal diagnosis. After birth, this syndrome was confirmed on the basis of growth restriction, long eyelashes, characteristic hair growth on the back, and hand abnormality (neonatal correlation on right). ${ }^{8,9}$

Figure 5. Pierre Robin sequence (case 2), 19.6 menstrual weeks. A, Another underdeveloped chin is suspected on 2DUS. The sagittal chin profile is not tapered but appears small and posteriorly displaced in relation to the maxilla. There is no evidence of a cleft lip or anterior hard palate defect from the axial view (bottom left, yellow arrow). B, Volume rendering allows head rotation for further examination of this region of interest. The left image shows an oblique view of the chin that could be mistakenly interpreted as normal. Rotation to the complete lateral view (right image) makes it easier to see a small chin. A defective posterior hard palate was also found at birth.

A

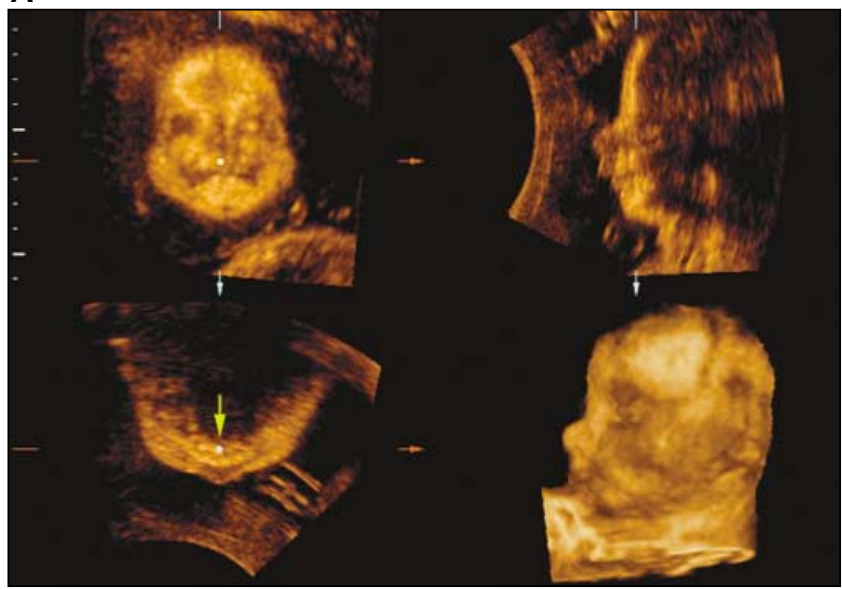

B

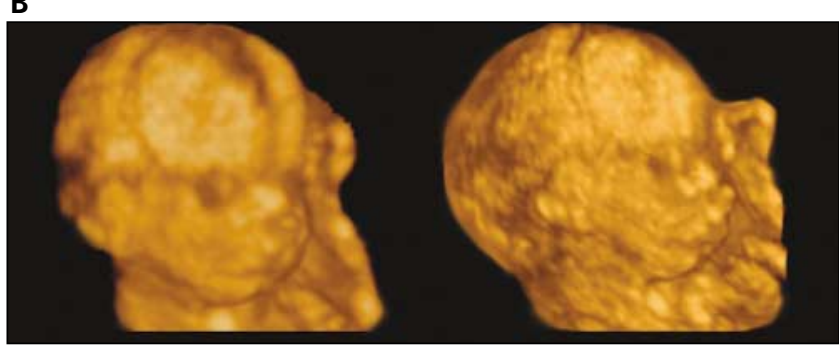

Figure 6. Pierre Robin sequence (case 3). Both the mother and her other daughter (top right) had mild underdevelopment of the chin. The 3DUS results (28.3 menstrual weeks) were correlated with the neonate (top left and middle). The final diagnosis was micrognathia with a posterior cleft palate defect as part of Pierre Robin sequence. Although a familial component was suspected, Stickler syndrome was unlikely because of lack of hearing and joint problems among other family members.

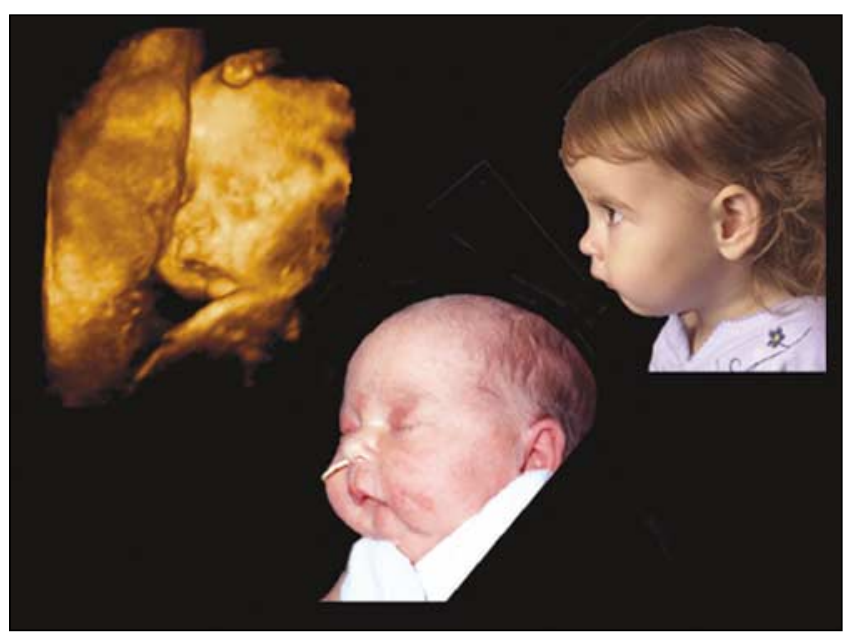




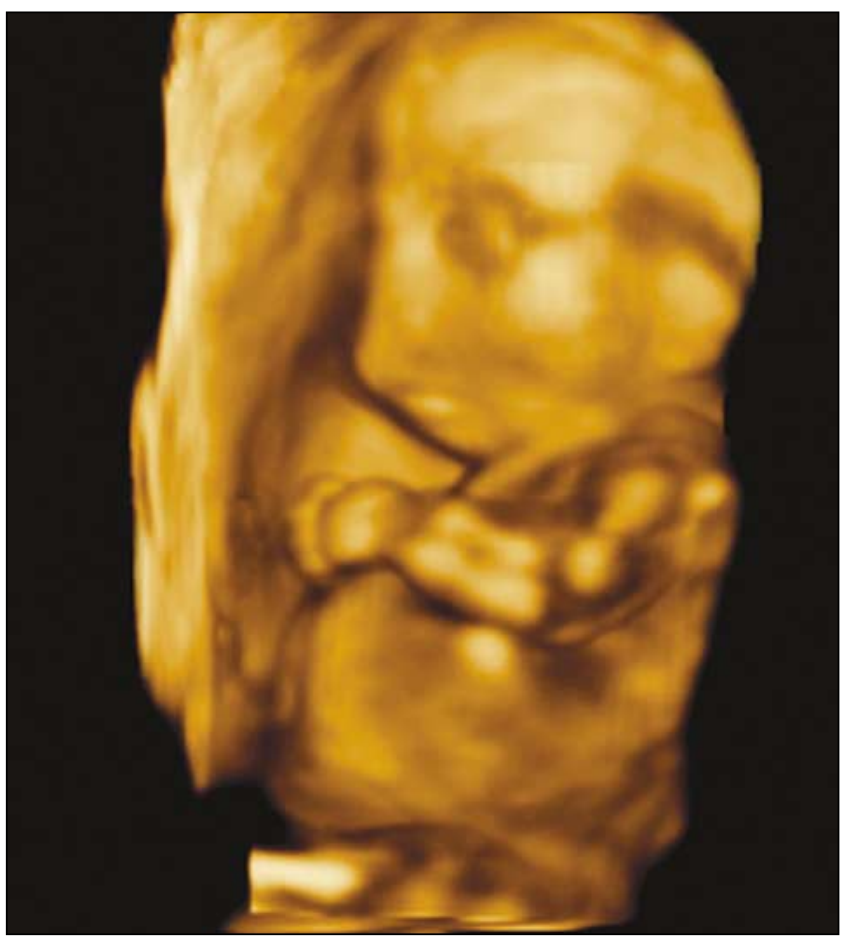

A

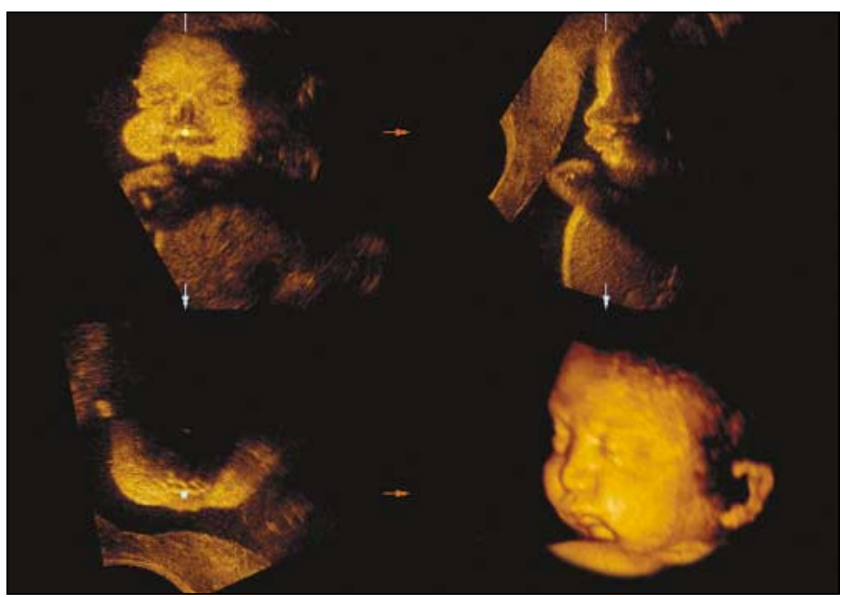

B

Figure 7. A, Hypochondrogenesis type II, 19.6 menstrual weeks. This fetus has micrognathia with shortened long bones and bilateral clubfeet. Note the disproportionately small trunk in comparison with a prominent abdomen. B, Hypochondrogenesis type II, 29.9 menstrual weeks. With advancing pregnancy, a small chin (bottom right) became more apparent without cleft lip or palate (bottom left, reference dot). The amniotic fluid volume was markedly increased, and the distal spine was never well visualized by ultrasonography. Delivery occurred at 35 menstrual weeks because of preterm labor, and the neonate died of pulmonary hypoplasia shortly after birth. The final diagnosis was hypochondrogenesis type II, which is similar to achondrogenesis type II because of marked phenotypic variability. ${ }^{10}$

Figure 8. Severe micrognathia, 15.7 menstrual weeks. A, Severe micrognathia is associated with craniofacial dysmorphology, aglossia, and multiple skeletal abnormalities. Increased amniotic fluid volume allowed acquisition of high-quality volume data. B, Postnatal photograph of the stillborn male neonate (right) showing excellent correlation of dysmorphic facial features. The left upper extremity is absent and associated with the rudimentary presence of both femur bones. Part of the right upper extremity is also missing.

A

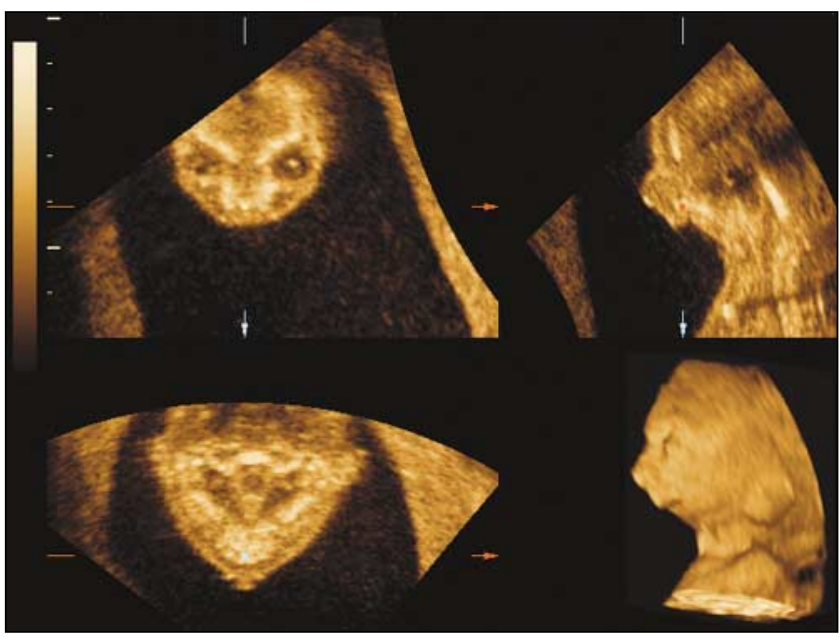

\section{B}

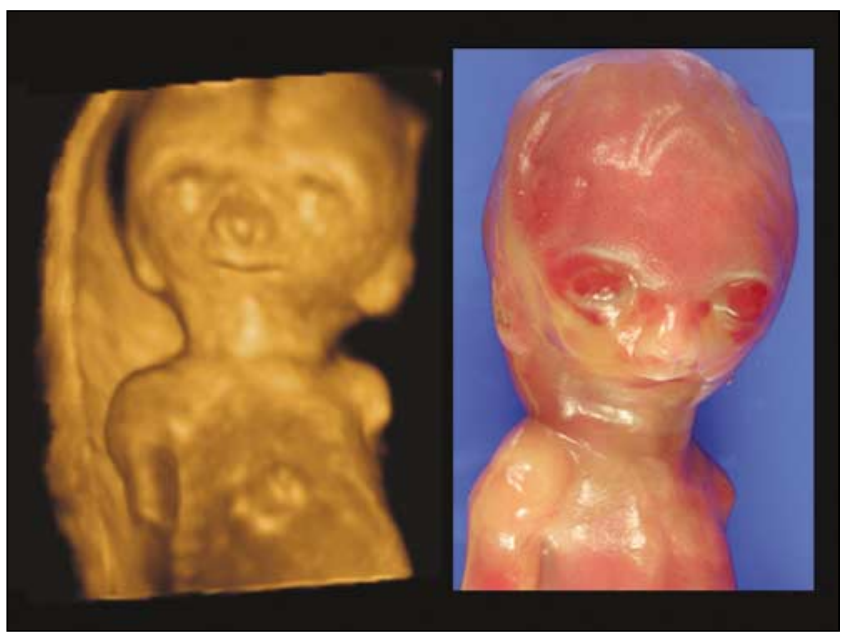




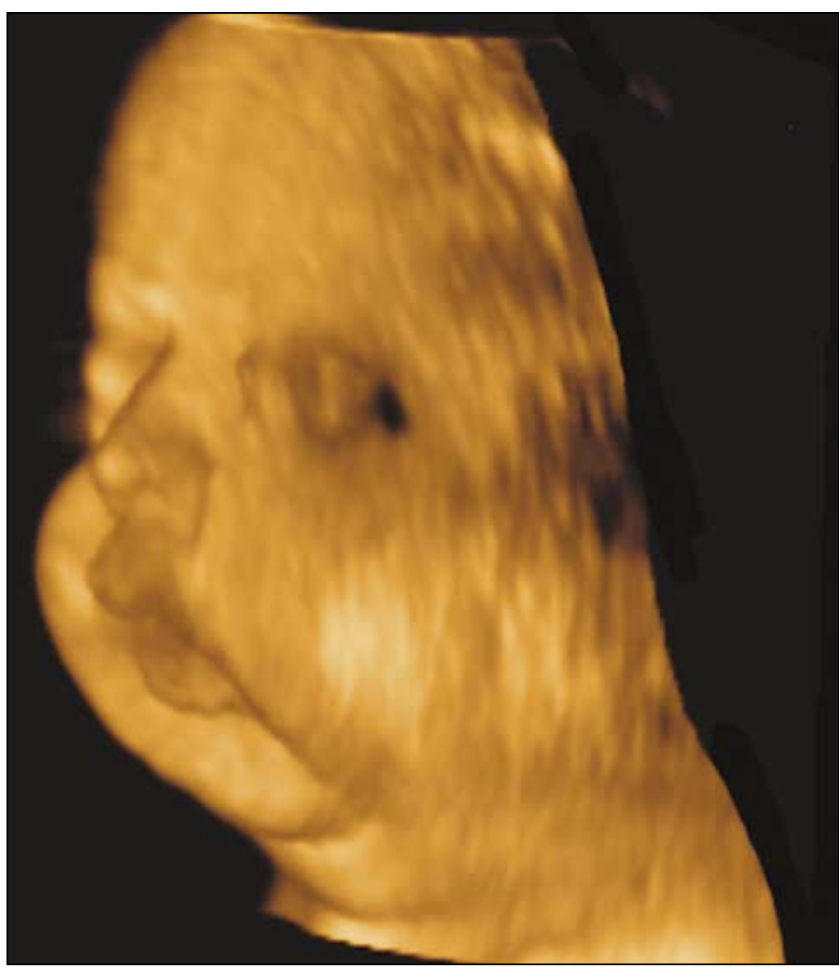

A

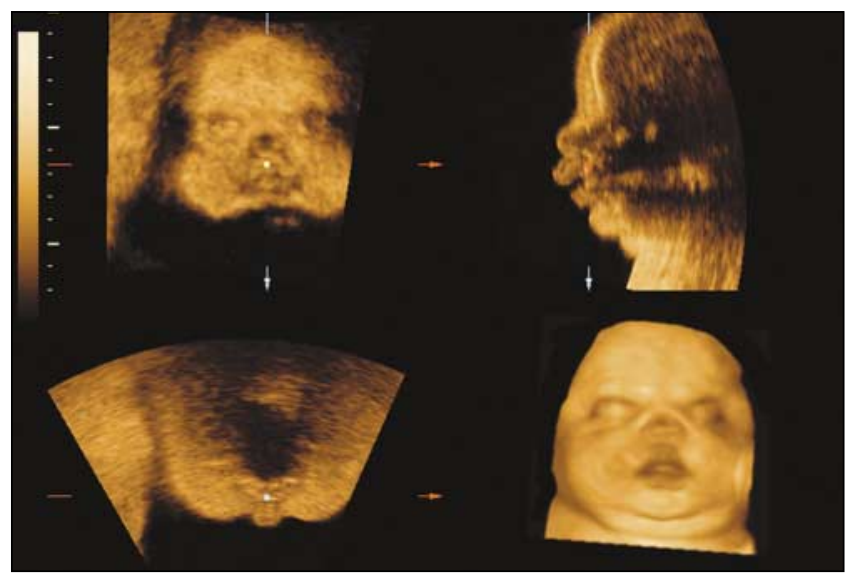

B

Figure 9. Caffey disease, 21.9 menstrual weeks. A, Delivery occurred at 36.6 menstrual weeks and was complicated by hydramnios. Surface rendering of the face initially suggested the presence of micrognathia. Postnatal examination confirmed Caffey disease with osteosclerosis that led to thickened clavicles and ribs. ${ }^{11}$ Severe expansion of the bowed long bones showed a cortical new bone formation. B, Despite the initial false impression of a small chin on surface rendering, a midline sagittal view (top right) provides evidence that the chin is actually well developed. The redundant soft tissue of the lips and cheeks $(\mathbf{A})$ can make the chin appear relatively small. Similar appearances of the chin can also be encountered in infants of diabetic mothers. This case emphasizes the importance of comparing both 3D multiplanar and surface-rendered images with the initial diagnostic impression on 2DUS.

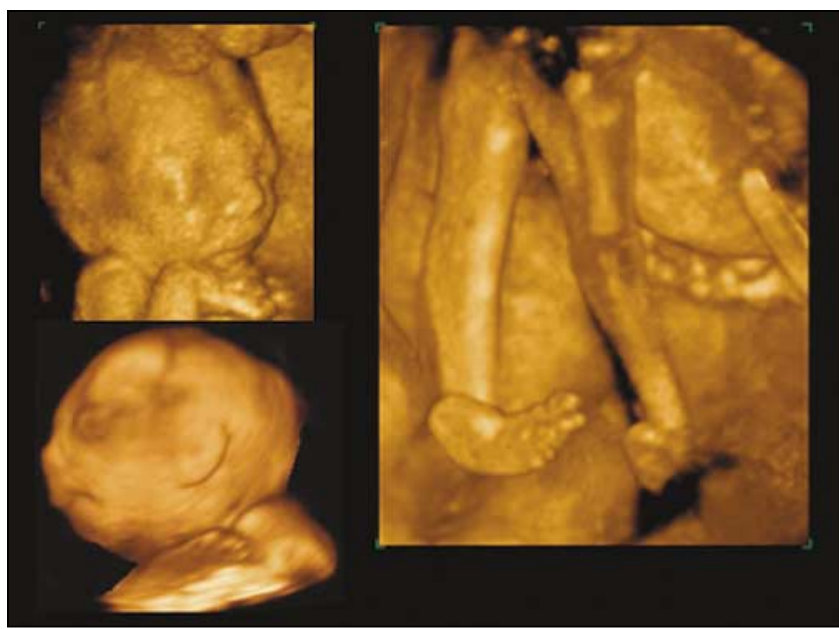

Figure 10. Micrognathia, 21.0 menstrual weeks. Surface rendering of the fetus is particularly well defined because of increased amniotic fluid volume. A small chin is evident when the fetal face is viewed from the side (bottom left). Multiple joint contractures, bilateral clubfeet, and lumbar spine scoliosis (not shown) are also present. The wrist, knee, and ankle joints are immobile. This neonate died 1 day after premature delivery at 30.4 menstrual weeks. The presumptive diagnosis was arthrogryposis, although an autopsy was not performed after delivery.

\section{References}

1. Online Mendelian Inheritance in Man. Bethesda, MD: National Center for Biotechnology Information, National Library of Medicine. Available at: http:// www.ncbi.nlm.nih.gov/omim.

2. Bromley B, Benacerraf BR. Fetal micrognathia: associated anomalies and outcome. J Ultrasound Med 1994; 13:529-533.

3. Nicolaides KH, Salvesen DR, Snijders RJM, Gosden CM. Fetal facial defects, associated malformations and chromosomal abnormalities. Fetal Diagn Ther 1993; 8:1-9.

4. Hsieh YY, Chang CC, Tsai HD, Yang TC, Lee CC, Tsai $\mathrm{CH}$. The prenatal diagnosis of Pierre-Robin sequence. Prenat Diagn 1999; 19:567-569.

5. Lee W, Kirk JS, Shaheen KW, Romero R, Hodges $\mathrm{AN}$, Comstock CH. Fetal cleft lip and palate detection by three-dimensional ultrasonography. Ultrasound Obstet Gynecol 2000; 16:314-320. 
6. Silverman FN, Strefling AM, Stevenson DK, Lazarus J. Cerebro-costo-mandibular syndrome. J Pediatr 1980; 97:406-416.

7. Megier $\mathrm{P}$, Ayeva-Derman M, Esperandieu O, Aubry MC, Couly G, Desroches A. Prenatal ultrasonographic diagnosis of the cerebro-costo-mandibular syndrome: case report and review of the literature. Prenat Diagn 1998; 18:1294-1299.

8. Jackson L, Kline AD, Barr MA, Koch S. de Lange syndrome: a clinical review of 310 individuals. Am J Med Genet 1993; 47:940-946.

9. Manouvrier $S$, Espinasse $M$, Vaast $P$, et al. Brachmann-de Lange syndrome: pre- and postnatal findings. Am J Med Genet 1996; 62:268-273.

10. Borochowitz Z, Ornoy A, Lachman R, Rimoin DL. Achondrogenesis II, hypochondrogenesis: variability versus heterogeneity. Am J Med Genet 1986; 24: 273-288.

11. Lecolier B, Bercau G, Gonzales M, et al. Radiographic, haematological, and biochemical findings in a fetus with Caffey disease. Prenat Diagn 1992; 12:637-641.

12. Pilu B, Reece EA, Romero R, Bovicelli L, Hobbins JC. Prenatal diagnosis of craniofacial malformations with ultrasonography. Am J Obstet Gynecol 1986; 155:45-50.

13. Otto C, Platt LD. The fetal mandible measurement: an objective determination of fetal jaw size. Ultrasound Obstet Gynecol 1991; 1:12-17.

14. Chitty LS, Campbell S, Altman DG. Measurement of the fetal mandible: feasibility and construction of a centile chart. Prenat Diagn 1993; 13:749-756.

15. Paladini D, Morra T, Teodoro A, Lamberti A, Tremolaterra F, Martinelli P. Objective diagnosis of micrognathia in the fetus: the jaw index. Obstet Gynecol 1999; 93:382-386.

16. van der Haven I, Mulder JW, van der wal KGH, Hage JJ, de Lange-de Klerk ESM, Hauman TJ. The jaw index: new guide defining micrognathia in newborns. Cleft Palate Craniofac J 1997; 34:240-241.

17. Rotten $D$, Levaillant JM, Martinez $H$, Ducou le Pointe $H$, Vicaut $E$. The fetal mandible: a $2 \mathrm{D}$ and $3 \mathrm{D}$ sonographic approach to the diagnosis of retrognathia and micrognathia. Ultrasound Obstet Gynecol 2002; 19:122-130. 